\title{
Lactic Acidosis as an Unusual Finding in Undifferentiated Carcinoma
}

\author{
Adrian Viteri-Noel ${ }^{a} \quad$ Maria Eugenia Cobo-Reinoso ${ }^{a}$ \\ Blanca de Felipe-Noguerales ${ }^{b}$ Luis Manzano ${ }^{a, c}$ \\ aInternal Medicine Department, Hospital Universitario Ramón y Cajal, IRYCIS, Madrid, Spain; \\ bHaematology Department, Hospital Universitario Ramón y Cajal, IRYCIS, Madrid, Spain; \\ 'Faculty of Medicine, Universidad de Alcalá (UAH), Madrid, Spain
}

\section{Keywords}

Undifferentiated carcinoma · Lactic acidosis · Differential diagnosis

\begin{abstract}
Lactic acidosis associated with solid neoplasms is a rare complication; its occurrence is poorly described, and it is associated with a poor prognosis. We present the case of an 84-year-old woman who was admitted to the internal medicine department with a diagnosis of urinary tract infection accompanied by a blood gas analysis showing lactic acidosis. During her admission, an abdominal mass was evidenced and finally diagnosed as undifferentiated carcinoma. We wanted to emphasize the importance of correlating clinical and laboratory data at the time of making a diagnostic approach and also highlight other possible explanations of lactic acidosis that should be considered in addition to hypoperfusion due to sepsis, especially in the elderly who are at increased risk of malignancy.
\end{abstract}

\section{Introduction}

Lactic acidosis is the most common type of metabolic acidosis found in hospitalized patients. Lactic acid may be overproduced in cases of increased anaerobic metabolism or decreased metabolic clearance due to a mitochondrial impairment [1]. Hypoperfusion is one of the most common causes of this finding, and this in turn can be caused by different pathologies such as hemorrhage or sepsis. However, there are other possible explanations for the increase of this metabolite [2]. Since malignancy may be a treatable cause, it is important to include it in the differential diagnosis [3]. On the other hand, urinary tract infections are one of the most frequent causes of emergency consultations, and older age is associated with a 
worse outcome, increasing the risk of sepsis [4]. As lactic acidosis can be associated with both (malignancy and sepsis), it is therefore important to take a good clinical history, physical examination, and complementary tests to guide diagnosis and treatment.

\section{Case Report}

An 84-year-old woman was admitted to the emergency department complaining of urinary hesitancy and itching during micturition. The patient had no medical history, was independent for all activities of daily living, and was not taking any medication prior to admission. She had no history of smoking or alcohol consumption. The patient had a 2-week history of poor general condition with asthenia and anorexia.

On physical examination, she was normotensive $(136 / 75 \mathrm{~mm} \mathrm{Hg})$ with a heart rate of 64 beats per minute and afebrile. Her neurological, cardiovascular, pulmonary, abdominal, and dermatological examinations were apparently normal.

The admission complete blood count reported a normocytic, normochromic anemia of $9.5 \mathrm{~g} / \mathrm{dL}(11.8-14.8 \mathrm{~g} / \mathrm{dL})$, platelets of $338 \times 10^{3} / \mu \mathrm{L}\left(140-440 \times 10^{3} / \mu \mathrm{L}\right)$, and mild leukocytosis of $12.40 \times 10^{3} / \mu \mathrm{L}\left(3.90-11.1 \times 10^{3} / \mu \mathrm{L}\right)$ with a neutrophil predominance of $83.5 \%$. In biochemistry, a stage II chronic kidney disease (glomerular filtration rate of $68 \mathrm{~mL} / \mathrm{min}$ ) associated with a natremia level of $135 \mathrm{mmol} / \mathrm{L}(135-145 \mathrm{mmol} / \mathrm{L})$ and a potassium level of $4.1 \mathrm{mmol} / \mathrm{L}$ (3.5-4.5 mmol/L) was found. Liver function showed a mild hypoalbuminemia of $2.47 \mathrm{~g} / \mathrm{dL}(3.50-5.00 \mathrm{~g} / \mathrm{dL})$ with normal total bilirubin, elevated GGT, alkaline phosphatase, and LDH of $92 \mathrm{U} / \mathrm{L}$ (7-30 U/L), $229 \mathrm{U} / \mathrm{L}$ (42-141 U/L), and $261 \mathrm{U} / \mathrm{L}(140-240 \mathrm{U} / \mathrm{L})$, accordingly. The C-reactive protein level was $212.5 \mathrm{mg} / \mathrm{dL}(0.0-5.0 \mathrm{mg} / \mathrm{dL})$ with a procalcitonin level of $0.52 \mathrm{ng} / \mathrm{mL}$. Hemostasis was within the normal range. Venous blood gas showed $\mathrm{pH}$ 7.44 (normal = 7.35-7.45), $\mathrm{pCO}_{2} 30 \mathrm{~mm} \mathrm{Hg}$ (normal = 35-45 mm Hg), $\mathrm{pO}_{2} 23 \mathrm{~mm} \mathrm{Hg}$ (normal $=80-100 \mathrm{~mm} \mathrm{Hg}$ ), $\mathrm{pHCO}_{3} 20.4 \mathrm{mmol} / \mathrm{L}$ (normal = 23-29 mmol/L), and a lactic acid level of $6.10 \mathrm{~mm} / / \mathrm{L}(0.45-1.9 \mathrm{~mm} / / \mathrm{L})$.

With all these data, antibiotics and fluid therapy were started, and the patient was admitted to the internal medicine department with a diagnosis of urinary tract infection with possible signs of early sepsis. Once on the internal medicine department, the patient was reassessed, and on abdominal examination, an epigastric mass was palpated.

Urine culture results revealed the growth of a poly-susceptible Escherichia coli. With these results, a complicated urinary tract infection was identified; however, the clinical situation did not correlate with the laboratory findings of early sepsis, since the patient was hemodynamically stable without any signs of hypoperfusion.

In order to expand the diagnosis, a body CT scan was requested. Findings were compatible with a $9.5 \times 8 \mathrm{~cm}$ mass in the root of the mesentery of uncertain organ dependence, with signs of peritoneal carcinomatosis and secondary liver metastasis (shown in Fig. 1) associated with probable signs of portal thrombosis. The patient was informed, and due to her previous good quality of life and good general condition, it was decided to continue with the diagnosis by performing an image-guided gross biopsy. Five days after admission, following completion of antibiotics, a new blood test showed an increase in C-reactive protein $(296.6 \mathrm{mg} / \mathrm{dL})$ and compensated metabolic acidosis illustrated by an arterial blood gas with a $\mathrm{pH}$ of $7.40, \mathrm{pCO}_{2}$ of $32 \mathrm{~mm} \mathrm{Hg}, \mathrm{pO}_{2}$ of $91 \mathrm{~mm} \mathrm{Hg}, \mathrm{pHCO}_{3}$ of $19.8 \mathrm{mmol} / \mathrm{L}$, and an increased lactic acid level of $10.4 \mathrm{~mm} / \mathrm{L}(0.45-1.90 \mathrm{~mm} / \mathrm{L})$.

However, the patient remained stable with a blood pressure of around 130/80 $\mathrm{mm} \mathrm{Hg}$, not tachycardic, with a good level of consciousness, and normal kidney function. Treatment with fluid therapy and bicarbonate was started with a partial correction on the acidosis. Likewise, new sets of urine and blood cultures were obtained and escalated to broad-spectrum

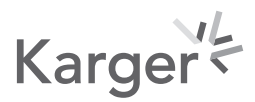




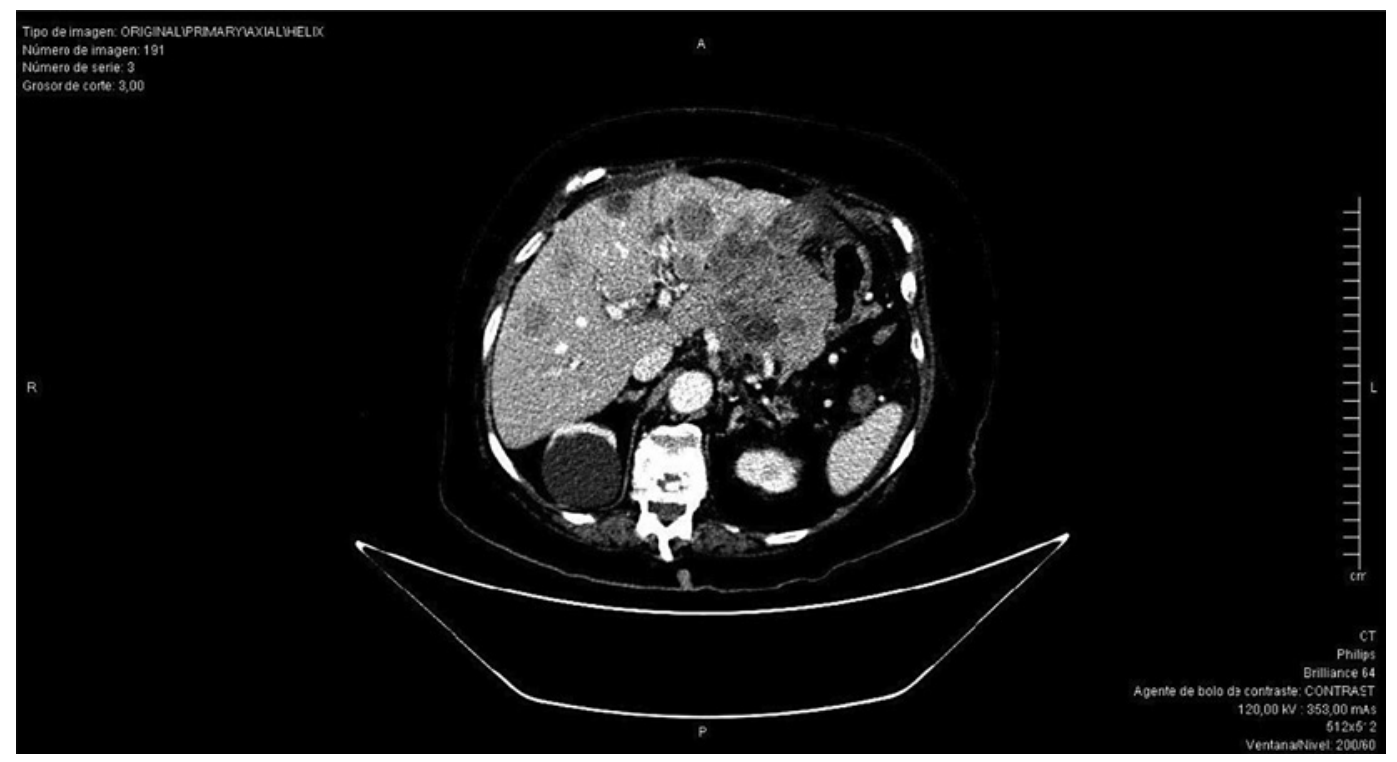

Fig. 1. A CT scan of the abdomen of the patient is shown. There is a mass in the root of the mesentery of approximately $9.5 \times 8 \mathrm{~cm}$, with a necrotic center, of dubious organ dependence. The lesion contacts the lesser curvature of the stomach, right hepatic lobe, and adjacent jejunal loops without a fatty separation plane. Countless hepatic lesions are suggestive of secondary deposit. Multiple nodular peritoneal soft tissue lesions compatible with carcinomatosis. Intraperitoneal free fluid of perihepatic, perisplenic distribution and in both flanks.

antibiotic therapy. After ruling out other causes of lactic acidosis since all cultures were informed as negative, we assumed a probable correlation with the malignancy.

After 2 days, suddenly the patient showed general discomfort and abdominal pain with signs of hypoperfusion accompanied by lower blood pressures $(70 / 50 \mathrm{~mm} \mathrm{Hg})$ and tachycardia (120 beats per minute). For this reason, a new CT scan was performed to exclude complications, which revealed significant growth of the mass, with signs of acute intratumoral bleeding and hematic content in the left retroperitoneal region. Growth of intraperitoneal implants in the left iliac fossa, with signs of infiltration, and ischemia of a segment of the transverse colon confirmed portal thrombosis compared to the previous scan (shown in Fig. 2). With all these findings, it was decided to proceed to symptomatic treatment, and the patient died within a few hours. Pathology findings were informed a few days later compatible with a poorly differentiated enteroid adenocarcinoma suggestive of colonic origin.

\section{Discussion}

This case report highlights the importance of considering the different causes of lactic acidosis when making a diagnostic approach. Lactic acidosis occurs normally due to hypoperfusion as a response of the cells to poor oxygen supply making them to change from an aerobic to an anaerobic metabolism [5]. But, its production can be explained by other situations. For this reason, lactic acid production has been classified into 3 different mechanisms [6]. Type A, which is the most common, is associated with a mismatch between oxygen supply and tissue oxygen consumption. This mechanism encompasses most common pathologies like respiratory, traumatological, and infectious. Type D is less common and is often correlated 


\section{Case Reports in Oncology}
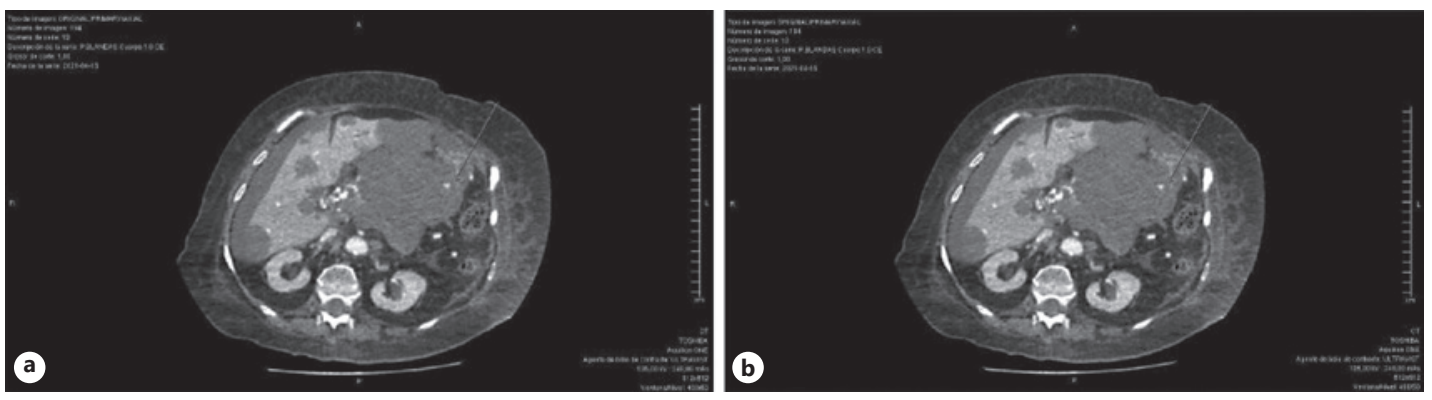

Fig. 2. An angio-CT scan of the patient is shown. a Significant growth of the solid mass centered in the pancreatic cell $(100 \times 124 \mathrm{~mm})$, which infiltrates the gastric lesser curvature, the antropyloric region, and the liver. It also presents contrast hypo-uptake with respect to previous CT, probably in relation to necrosis. b In the center of the mass, in its left lateral aspect, hyperdense foci, visualized only in the portal phase, suggests intratumoral bleeding foci.

with poor clearance of lactic acid as can occur in short bowel syndrome. Malignancy-related lactic acidosis has been described as part of type B lactic acidosis which includes diseases that impair cellular metabolism induced by toxins and areas of ischemia [6]. Other common diseases can produce type B lactic acidosis like diabetes mellitus (metformin-induced lactic acidosis), alcoholism, and HIV infection (antiretroviral-induced mitochondrial dysfunction) $[7,8]$. It is important to note that these types of acidosis can occur at the same time in the same patient given by different pathways in the same pathology [9].

Although lactic acidosis is not a common finding in malignancies, it is associated with an increased risk of mortality and a need for urgent chemotherapy [5]. It has been more often described in cases of hematologic malignancies as these diseases are related to high cell turnover although cases have also been described in solid tumors $[5,10]$.

There are different pathways that explain lactic acidosis in patients presenting solid tumors: first, probably tumor cells change their metabolism to favor glycolysis and production of lactic acid from pyruvate known as the Warburg effect $[10,11]$. An increase in hexokinase II and phosphofructokinase has been described in these cells, which promotes the glycolysis pathway and generation of lactic acid [12]. Liver metastasis could also contribute to lactic acid production via an impaired liver function. Another explanation is associated with the relationship of a fast-growing neoplasm and poor vascularity around [13].

There are few cases of solid tumors associated with lactic acid in the literature. The revision made by Liu et al. [14] showed a set of 50 cases of lactic acidosis associated with solid tumors and 14 cases since 2010. These tumors share common characteristics, and a lot of them were poorly differentiated and had liver metastasis.

Although on admission, lactic acid elevation was interpreted as due to urinary sepsis, she never presented clinical signs that would justify this process. Likewise, despite having received a targeted antibiotic, lactic acid continued to increase.

This is a case in which we concluded that the lactic acidosis presented by the patient from the beginning was due to advanced malignant disease. And, it serves to illustrate the importance of making a good correlation between clinical and laboratory findings.

\section{Acknowledgments}

We gratefully acknowledge all internal medicine clinicians from Hospital Universitario Ramón y Cajal for their assistance in the process of writing the manuscript. 


\section{Case Reports in Oncology}

\begin{tabular}{l|l}
\hline Case Rep Oncol 2021;14:1237-1241 \\
\hline DOI: 10.1159/000517853 & $\begin{array}{l}\text { @ 2021 The Author(s). Published by S. Karger AG, Basel } \\
\text { www.karger.com/cro }\end{array}$ \\
\hline
\end{tabular}

Viteri-Noel et al.: Differential Diagnosis of Lactic Acidosis in an Elderly Patient

\section{Statement of Ethics}

The study is exempt from ethics committee approval as it is a presentation of a case, and a nondrug trial was involved. Written informed consent was obtained from the patient's daughter for publication of this case report.

\section{Conflict of Interest Statement}

The authors have no conflicts of interest to declare.

\section{Funding Sources}

There were no funding sources for development of this manuscript.

\section{Author Contributions}

All authors have contributed equally to the development of this manuscript.

\section{Data Availability Statement}

Data that support these findings are available upon request to the correspondent author.

\section{References}

1 Adeva-Andany M, López-Ojén M, Funcasta-Calderón R, Ameneiros-Rodríguez E, Donapetry-García C, Vila-Altesor $\mathrm{M}$, et al. Comprehensive review on lactate metabolism in human health. Mitochondrion. 2014;17:76-100.

2 El Imad T, El Khoury L, Geara AS. Warburg's effect on solid tumors. Saudi J Kidney Dis Transpl. 2014;25(6):1270-7.

3 Liu QS, Harji F, Jones A, Tarnower AC. Type B lactic acidosis: a rare oncological emergency. BMJ Case Rep. 2020;13(3):e233068.

4 Foxman B. Urinary tract infection syndromes: occurrence, recurrence, bacteriology, risk factors, and disease burden. Infect Dis Clin North Am. 2014;28(1):1-13.

5 Conceição MS, Gáspari AF, Ramkrapes APB, Junior EMM, Bertuzzi R, Cavaglieri CR, et al. Anaerobic metabolism induces greater total energy expenditure during exercise with blood flow restriction. PLoS One. 2018;13(3): e0194776.

6 Foucher CD, Tubben RE. Lactic Acidosis. In: StatPearls. Treasure Island (FL): StatPearls Publishing; 2021 Jan. Available from: https://www.ncbi.nlm.nih.gov/books/NBK470202/.

7 DeFronzo R, Fleming GA, Chen K, Bicsak TA. Metformin-associated lactic acidosis: current perspectives on causes and risk. Metabolism. 2016;65(2):20-9.

8 Calza L, Manfredi R, Chiodo F. Hyperlactataemia and lactic acidosis in HIV-infected patients receiving antiretroviral therapy. Clin Nutr. 2005;24(1):5-15.

9 Singh M, Ajmeri AN, Suliman MS, Zaheer K, Al-Astal AK. A challenging case of coexisting type A and type B lactic acidosis: a case report. Cureus. 2019;11(1):e3944.

10 Schuh AM, Leger KJ, Summers C, Uspal NG. Lactic acidosis in a critically ill patient: not always sepsis. Pediatr Emerg Care. 2018;34(9):e165-7.

11 Vander Heiden MG, Cantley LC, Thompson CB. Understanding the Warburg effect: the metabolic requirements of cell proliferation. Science. 2009;324(5930):1029-33.

12 Tanner LB, Goglia AG, Wei MH, Sehgal T, Parsons LR, Park JO, et al. Four key steps control glycolytic flux in mammalian cells. Cell Syst. 2018;7(1):49-e8. e8

13 de Groot R, Sprenger RA, Imholz AL, Gerding MN. Type B lactic acidosis in solid malignancies. Neth J Med. 2011; 69(3):120-3.

14 Liu QS, Harji F, Jones A, Tarnower AC. Type B lactic acidosis: a rare oncological emergency. BMJ Case Rep. 2020;13(3):e233068. 13 